\title{
Endophyte production of reactive oxygen species is critical for maintaining the mutualistic symbiotic interaction between Epichloë festucae and Pooid grasses
}

\author{
A. TANAKA ${ }^{1}$, M.J. CHRISTENSEN ${ }^{2}$, D. TAKEMOTO ${ }^{1}$ and B. SCOTT ${ }^{1}$ \\ ${ }^{I}$ Centre for Functional Genomics, Institute of Molecular BioSciences, \\ Massey University, Private Bag 11 222, Palmerston North, New Zealand \\ ${ }^{2}$ AgResearch, Grasslands Research Centre, Tennent Drive, Private Bag 11 008, Palmerston North New Zealand
}

d.b.scott@massey.ac.nz

\begin{abstract}
Perennial ryegrass, tall fescue and meadow fescue infected with a nox $A$ mutant defective in the ability to produce reactive oxygen species (ROS), had a stunted phenotype and showed premature senescence. Microscopic observations of leaf tissue from perennial ryegrass and tall fescue showed a lack of regulated growth of mutant hyphae in these tissues with increasedv branching, growth and vascular colonisation. However, the fungal phenotype of nox $A$-infected meadow fescue was very similar to the wild-type phenotype suggesting that an increase in fungal biomass and vascular bundle colonisation is not necessarily a requirement for host stunting. An alternative hypothesis to explain the stunting phenotype is a breakdown in normal signaling between endophyte and host that is ROS dependent.
\end{abstract}

Figure 1 Phenotype of $E$. festucae wild-type strain $\mathrm{Fl} 1$ in different grasses. Light micrographs of transverse sections of the outer leaf sheath of perennial ryegrass (A), tall fescue (B) and meadow fescue (C) infected with Fl1, and uninfected leaf (D), stained with toluidine blue. Hyphae of E. festucae are indicated by arrowheads. Xy, xylem; Ph, phloem. Bars $=20 \mu \mathrm{m}$.
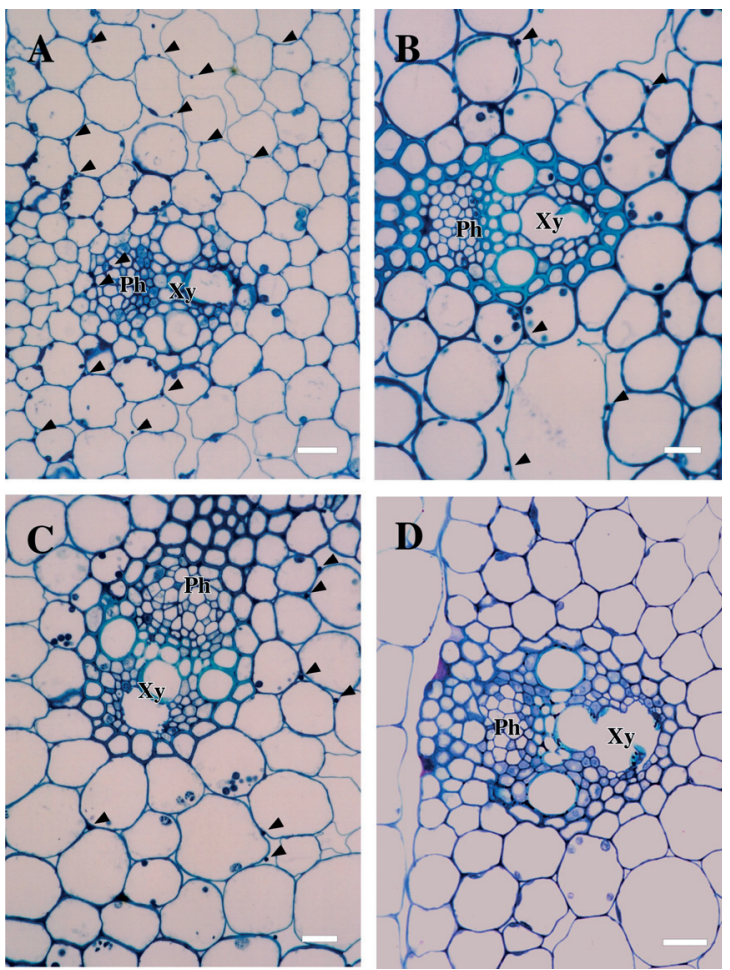

Keywords: Epichloë festucae, Lolium perenne, NoxA, NADPH oxidase, reactive oxygen species (ROS)

\section{Introduction}

Fungal endophyte production of reactive oxygen species (ROS) is critical for maintaining a mutualistic symbiotic interaction between Epichloë festucae and the grass host Lolium perenne (Tanaka et al. 2006). In wild-type associations, E. festucae grows systemically in the intercellular spaces of vegetative and reproductive tissues as infrequently branched hyphae parallel to the axis of the leaf (Tan et al. 2001; Christensen et al. 2002). Growth of the hyphae is strictly intercellular and synchronised with that of the host grass throughout the life cycle of the plant. Inactivation of a gene encoding a specific NADPH oxidase

Figure 2 Phenotype of $E$. festucae noxA mutuant strain FR2 in perennial ryegrass. (A) Light micrograph of transverse section of the outer leaf sheath stained with toluidine blue. Hyphae of $E$. festucae are indicated by arrowheads. Xy, xylem; Ph, phloem. Bars $=20 \mu \mathrm{m}$. (B) to (D) Transmission electron micrographs of cross sections of the outer leaf sheath. Bars $=2 \mu \mathrm{m}$ in (B) to (D).
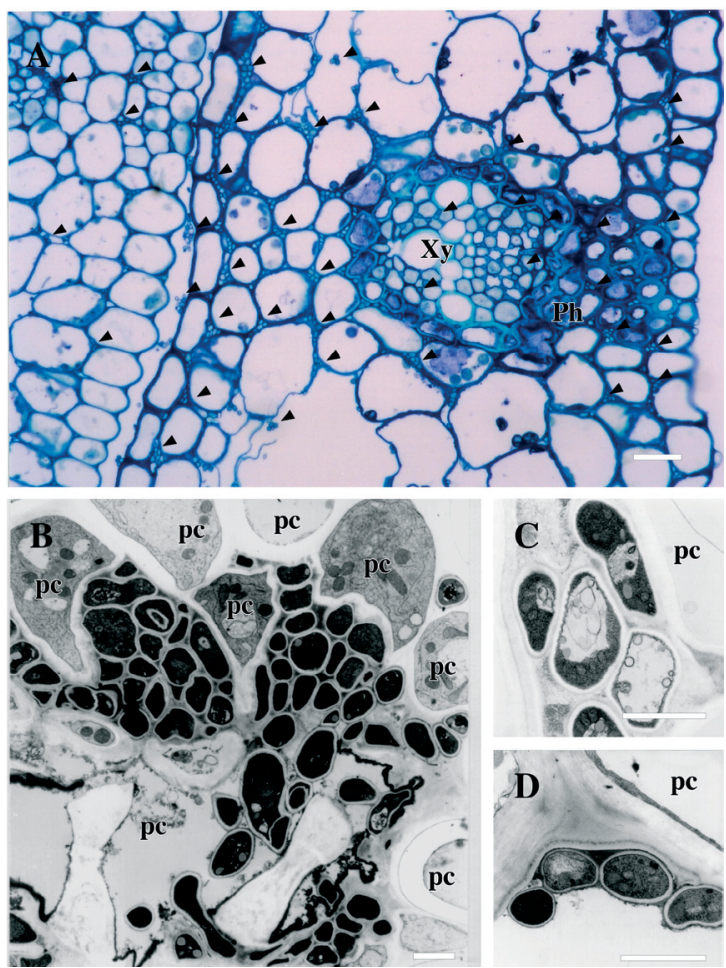
Figure 3 Phenotype of E. festucae noxA mutuant strain FR2 in tall fescue. (A) Light micrograph of transverse section of the leaf blade stained with toluidine blue. Hyphae of $E$. festucae are indicated by arrowheads. Xy, xylem; Ph, phloem. Bars $=20 \mu \mathrm{m}$. (B) to (D) Transmission electron micrographs of cross sections of the leaf blade (B) and outer leaf sheath (C) and (D). Bars $=2 \mu \mathrm{m}$ in (B) and (D), and $1 \mu \mathrm{m}$ in (C).
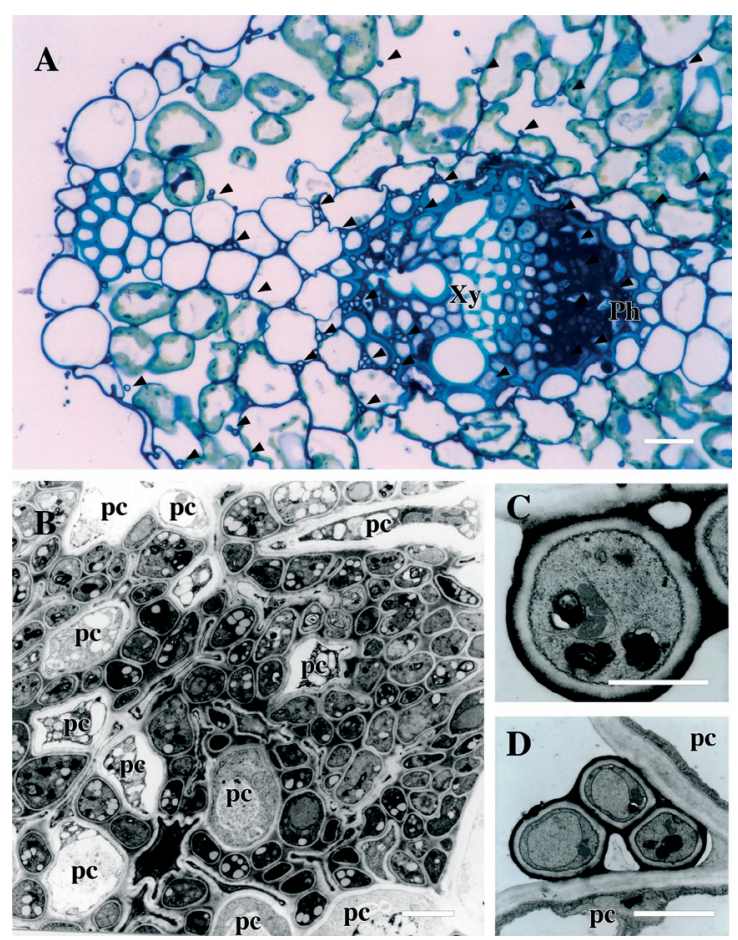

isoform, NoxA, by plasmid insertional mutagenesis, resulted in unregulated growth of the fungal hyphae in vegetative tissue and premature senescence of the host plant (Tanaka et al. 2006). Light microscopic analysis of cross sections of leaf tissue showed that large numbers of hyphae were present in older leaves of plants infected with the noxA mutant, with extensive colonisation of the vascular bundles, including both phloem and xylem. Transmission electron microscopy analysis of hyphae in the outer leaf revealed that mutant cells were frequently irregular in shape and highly vacuolated. Using electron microscopy to locate deposits of electron-dense cerium perhyroxides we were able to show that $\mathrm{H}_{2} \mathrm{O}_{2}$ production was significantly reduced in the endophyte extracellular matrix and associated plant cell walls of meristematic tissue infected with the nox $A$ mutant compared to wild-type.

The objective of this study was to further understand why endophyte production of ROS is so critical for E. festucae to maintain a mutualistic symbiotic interaction with its grass hosts by using both light and transmission electron microscopy to examine the interaction of the nox $A$ mutant with perennial ryegrass, tall fescue and meadow fescue.

\section{Methods}

Fungal strains and growth conditions

Cultures of Epichloë festucae wild-type strain F11 and noxA
Figure 4 Phenotype of $E$. féstucae noxA mutuant strain FR2 in meadow fescue. (A). Light micrograph of transverse section of the leaf blade with toluidine blue. Hyphae of E. festucae are indicated by arrowheads. Xy, xylem; Ph, phloem. Bars $=20 \mu \mathrm{m}$. (B) and (C) Transmission electron micrographs of cross sections of the leaf blade. Bars $=2$ $\mu \mathrm{m}$ in (B) and (C).
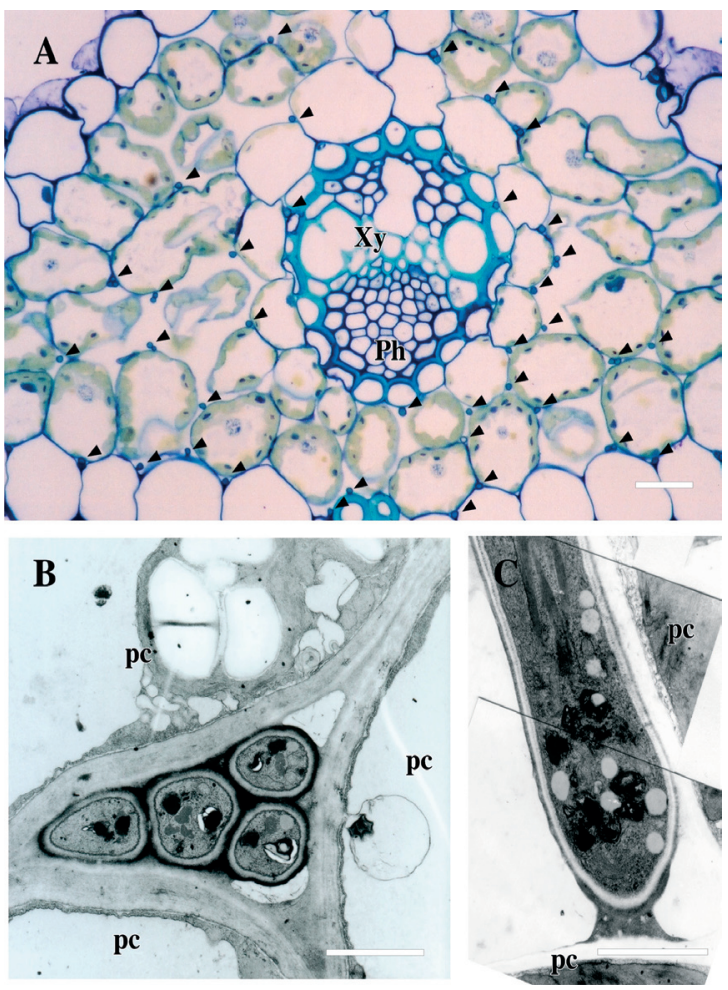

mutant strain FR2 (Tanaka et al. 2006) were grown on $2.4 \%$ potato dextrose (PD) agar at $22^{\circ} \mathrm{C}$.

Plant growth and endophyte inoculation conditions

Inoculation of endophyte-free seedlings of perennial ryegrass (Lolium perenne), tall fescue (Festuca arundinacea) and meadow fescue (Festuca pratensis) with E. festucae was performed by the method of Latch \& Christensen (1985). Plants were grown and tested for the presence of endophyte as described previously (Tanaka et al. 2005).

\section{Microscopy}

Small leaf pieces, 1-2 mm thick, from endophyte infected plant tissues were fixed in 3\% glutaraldehyde and $2 \%$ formaldehyde in $0.1 \mathrm{M}$ phosphate buffer ( $\mathrm{pH}$ 7.2) and then prepared for light microscopy and transmission electron microscopy as described by Spiers \& Hopcroft (1993). For light microscopy, the sections were stained with toluidine blue as described by Christensen et al. (2002). A Philips 201C transmission electron microscope was used to examine hyphal structure.

\section{Results}

Light microscopic analysis of toluidine blue-stained cross sections of leaf sheath (pseudostem) from perennial ryegrass (Fig. 1A), tall fescue (Fig. 1B) and meadow fescue (Fig. 1C) infected 
with wild-type strain F11 showed that hyphae were solitary, predominantly located in the intercellular spaces of the mesophyll tissue attached to plant cell walls, and cytoplasmically dense. Occasionally, hyphae were observed in vascular bundles of both young and old plant tissue (Fig. 1C). No hyphae were observed in uninfected plants (Fig. 1D). In contrast, perennial ryegrass plants infected with the noxA mutant, strain FR2, contained large numbers of vacuolated hyphae in the mesophyll tissue, with extensive colonisation of the vascular bundles, including both xylem and phloem (Fig. 2A). Transmission electron microscopy revealed that mutant cells were very abundant and frequently irregular in shape (Fig. 2B), sometimes contained large vacuoles (Fig. 2C), and lacked crystalline aggregations (Fig. 2D), that are typically found in wild-type cells. Occasionally, hyphae with wild-type morphology were observed in leaf tissue infected with the nox $A$ mutant and on one leaf sheath we observed hyphae with the unregulated nox $A$ growth phenotype on one side of the sheath and hyphae with wild-type regulated growth on the other side of the sheath. Furthermore, we observed that noxAinfected plants had increased susceptibility to powdery mildew (Blumeria graminis). As previously reported, perennial ryegrass plants infected with the nox $A$ mutant were stunted in appearance compared to wild-type and underwent premature senescence (Tanaka et al. 2006).

Tall fescue plants infected with the noxA mutant contained large numbers of vacuolated hyphae in the mesophyll leaf blade tissue, with extensive colonisation of the vascular bundles, including both xylem and phloem (Fig. 3A). Some hyphae in the mesophyll tissue were cytoplasmically dense and retained synchronised growth with the host plant. Transmission electron microscopy revealed that mutant cells were considerably greater in number than wild-type, were full of lipid droplets (Fig. 3B), and in contrast to perennial ryegrass, frequently contained crystalline aggregations (Figs. 3C \& 3D). Tall fescue plants infected with the nox $A$ mutant showed a stunted phenotype and underwent premature senescence compared to wild-type (Tanaka et al. 2006).

While meadow fescue plants infected with the nox $A$ mutant had a stunted phenotype (Tanaka et al. 2006), the concentration of hyphae in leaf blade mesophyll tissue, as observed by light microscopy, was only slightly different to that of the wildtype strain. Hyphae were cytoplasmically dense but frequently occurred in pairs. Very little colonisation of the vascular bundle was observed in this endophyte-grass host interaction. Transmission electron microscopy revealed that mutant cells frequently had protein crystal aggregates, similar in frequency and appearance to wild-type cells (Fig. 4B). In some cases hyphae had a wild-type phenotype, containing both lipid droplets and protein crystal aggregates (Fig. 4C).

\section{Discussion}

This work demonstrates that endophyte production of ROS is critical in maintaining mutualistic symbiotic interactions between E. festucae and perennial ryegrass, tall fescue or meadow fescue. In each host association, disruption of nox $A$ led to a stunting phenotype characterised by increased tillering and premature senescence of the host (Tanaka et al. 2006). The increased susceptibility of these plants to powdery mildew infection was indicative of the stressed state of the nox $A$-infected plants. However, the fungal phenotype associated with each of these mutant associations was variable. Hyphae of the nox $A$ mutant in association with either perennial ryegrass or tall fescue associations, showed increased colonisation of leaf sheath and blade tissue, due to increased hyphal branching and growth in the meristematic tissue, as well as ongoing growth following leaf maturation. In both associations there was extensive endophyte colonisation of the vascular bundle tissue, a phenotype previously observed for a natural isolate of E. festucae, strain E365, and ascospore progeny of a cross between this strain and E189, when inoculated into perennial ryegrass (Christensen et al. 1997). In contrast the noxA mutant in association with meadow fescue was very similar to the wild-type phenotype, showing little increase in the number of hyphae found in the leaf sheath and blade tissue and little colonisation of the vascular bundles. These results indicate that an increase in fungal biomass and vascular bundle colonisation are not necessary to induce the host stunting and premature senescence phenotype observed in all three nox $A$ associations. This is supported by the observation that ryegrass with L. multiflorum characteristics infected with Neotyphodium lolii strain AR1 show extensive colonisation of the vascular bundles but no stunting (Christensen et al. 2001). An alternative hypothesis to explain the stunting phenotype is a breakdown in wild-type signalling between endophyte and host, that is ROS dependent. The importance of ROS signalling is supported by the recent demonstration that disruption of gene encoding a Nox activator, noxR, also leads to a stunted phenotype when this mutant is introduced into perennial ryegrass (Takemoto et al. 2006). It will be of considerable interest to examine the hyphal phenotype of this mutant in associations with tall fescue and meadow fescue.

\section{ACKNOWLEDGEMENTS}

This research was supported by grant MAU0403 from the Royal Society of New Zealand Marsden Fund. The authors thank Andrea Bryant and Elizabeth Nickless (Massey University), and Douglas Hopcroft and Raymond Bennett (HortResearch) for technical assistance.

\section{REFERENCES}

Christensen, M.J.; Ball, O.J.P.; Bennett, R.J.; Schardl, C.L. 1997. Fungal and host genotype effects on compatibility and vascular colonization by Epichloë festucae. Mycological Research 101: 493-501.

Christensen, M.J.; Bennett, R.J.; Schmid, J. 2001. Vascular bundle colonization by Neotyphodium endophytes in natural and novel associations with grasses. Mycological Research 105: 1239-1245.

Christensen, M.J.; Bennett, R.J.; Schmid, J. 2002. Growth of Epichloë/Neotyphodium and p-endophytes in leaves of Lolium and Festuca grasses. Mycological Research 106: 93-106.

Latch, G.C.M.; Christensen, M.J. 1985. Artificial infection of grasses with endophytes. Annals of Applied Biology 107: 17-24.

Spiers, A.G.; Hopcroft, D.H. 1993. Black canker and leaf spot of Salix in New Zealand caused by Glomerella miyabeana (Colletotrichum gloeosporioides). European Journal of Forest Pathology 23: 92-102.

Tan, Y.Y.; Spiering, M.J.; Scott, V.; Lane, G.A.; Christensen, M.J.; Schmid, J. 2001. In planta regulation of extension of an endophytic fungus and maintenance of high metabolic rates in its mycelium in the absence of apical extension. Applied and Environmental Microbiology 67: 5377-5383.

Tanaka, A.; Tapper, B.A.; Popay, A.J.; Parker, E.J.; Scott, B. 2005. A symbiosis expressed non-ribosomal peptide synthetase from a mutualistic fungal endophyte of perennial ryegrass confers 
protection to the symbiotum from insect herbivory. Molecular Microbiology 57:1036-1050.

Tanaka, A.; Christensen, M.J.; Takemoto, D.; Park, P.; Scott, B. 2006. Reactive oxygen species play a role in regulating a fungus-perennial ryegrass mutualistic association. The Plant Cell 18: 1052-1066.
Takemoto, D.; Tanaka, A.; Scott, B. 2006. A p67 $7^{\text {Phox }-l i k e ~ r e g u l a t o r ~}$ is recruited to control hyphal branching in a fungal-grass mutualistic symbiosis. The Plant Cell 18: in press. 